Egyptian Journal of Aquatic Biology \& Fisheries

Zoology Department, Faculty of Science,

Ain Shams University, Cairo, Egypt.

ISSN $1110-6131$

Vol. 24(2): 225 - 237 (2020)

www.ejabf.journals.ekb.eg

\title{
Adaptability of the Nile Tilapia, Oreochromis niloticus Juveniles to Water Salinity by
} Controlling Dietary Sodium Chloride Levels

\author{
Abdelrhman M. Abdelrhman ${ }^{1}$, Zaki Z. Sharawy ${ }^{1}$, Ashraf M. A. S. Goda ${ }^{1}$, \\ Matthew J. Slater ${ }^{2}$ \\ ${ }^{1}$ Aquaculture Division, National of Oceanography \& Fisheries (NIOF), Cairo, Egypt \\ ${ }^{2}$ Alfred Wegener Institute for Polar and Marine Research, Bremerhaven, Germany \\ *Corresponding Author: abdelrhman_niof@hotmail.com
}

\section{ARTICLE INFO}

Article History:

Received: Feb. 16, 2020

Accepted: March 28, 2020

Online: April 1, 2020

Keywords:

Water Salinity

Sodium Chloride

growth

Feed utilization

$\mathrm{Na}^{+}$concentration

Nile tilapia

\begin{abstract}
Increasing groundwater scarcity and salinity pose challenges for high water demand aquaculture. Adapting animals to grow optimally under saline conditions is key to future growth in many important aquaculture regions. In the current study, 10-week controlled feeding experiment was carried out to investigate dietary sodium chloride supplementation $(10 \% \mathrm{NaCl})$ as a method of mitigating the effects of increasing water salinity $(0,10$ and 15 ppt) on growth and metabolism of juvenile Nile tilapia, Oreochromis niloticus in controlled feeding experiments. Fish fed diets supplemented with $10 \% \mathrm{NaCl}$ recorded significantly better weight gain when compared to fish fed control when held in 10 and 15 ppt salinity water over a period of ten weeks. Blood glucose and blood lactate concentrations were both significantly higher in fish fed diets supplemented with $10 \% \mathrm{NaCl}$ and increased with increasing water salinity. Fish fed diet containing $10 \% \mathrm{NaCl}$ recorded the highest values of $\mathrm{Na}^{+}$concentration in blood compared to other experimental groups. Feed utilization, as indicated by feed conversion ratio decreased significantly with water salinity $(\mathrm{P}<0.05)$. Feed intake also decreased with increasing water salinity. The obtained results indicated that salinity is a key factor in controlling growth of Nile tilapia. Results clearly indicate that negative effects of increased salinity in rearing water can be partially mitigated by salt inclusion in diets.
\end{abstract}

\section{INTRODUCTION}

Egypt is located in the subtropical belt and a major part of the country faces scarcity of rainfall, and thus significant water scarcity overall. Thus, it is extremely important to find possible alternatives for justified utilization of limited water resources. Requirements for water for agriculture and other urban activities has increased the pressure to develop aquaculture in brackish and seawater. Control of salt and water balance within a narrow limit is critical to life in all multicellular organisms, including teleost fishes. Salt tolerance is a term describing the overall fitness, or productivity, of the fish in a saline 
environment. It is a combination of different quantitative traits, such as metabolism, growth, osmoregulation, immunocompetence and fecundity (Cnaani and Hulata, 2011).

Nile Tilapia, O. niloticus belongs to family Cichlidae, which dominates freshwater fish culture and is an excellent candidate for aquaculture in brackish water due to their ability to tolerate a wide range of water salinity. Inter-specific variation in salinity tolerance may be used to select salt-tolerant species and develop salt-tolerant hybrids. Growth of $O$. niloticus at high salinity is significantly lower than that in freshwater Fineman, (1988), whereas survival is not affected by salinity. High salinity does seem to suppress, or at least delay, onset of reproduction in $O$. niloticus, thus presenting a practical method of population control to improve overall yields.

Recent studies by Appelbaum et al. (2008 a, 2008b) showed that gilthead sea bream juveniles reared in brackish water (3\% TDS) and fed a diet supplemented with $1.5 \%$ salt grew significantly $(\mathrm{P}<0.05)$ better than those fed the control diet (no added salt). A further study (Appelbaum and Arockiaraj, 2008 b) using diets supplemented with higher levels of salt (8,10 and 12\%) showed that gilthead sea bream juveniles reared in brackish water of $2.9 \%$ (TDS) salinity grew better and had the highest survival rate when fed a diet containing $12 \%$ salt. The salinity of the culture system is known to influence metabolism and homeostatic processes in fish, meaning the organism needs for nutrients and protein particularly, may differ in freshwater and saltwater systems (Altinok and Grizzle, 2001).

Yao et al. (2008) investigated the best conditions for transfer of Nile tilapia from freshwater to salt water. Nile tilapia fingerlings (8 to $12 \mathrm{~g}$ ) were transferred, either directly or gradually, from freshwater to water of variable salinities, and survival was monitored after 3 weeks. Survival of fish transferred directly to saline water was high (84.3\% to $96.8 \%$ ) until $17 \mathrm{ppt}$, but mortalities were significant (60-70\%) above that salinity. High rate of survival ( 78 to $81 \%$ ) was, however, achieved by gradual acclimation to salinity of $30 \mathrm{ppt}$ over two days. Larumbe-Moran et al. (2010) reported that culture of Nile tilapia is feasible in saline environments at up to $25 \mathrm{ppt}$ of salinity without affecting growth or survival range, and with no increases required in dietary protein if the standing biomass remains under critical maxima. Nonetheless, the tilapia do require higher protein intake at higher salinities to produce growth rates equivalent to those observed in freshwater environments. The present study was undertaken to determine the effects of water salinity and dietary supplementation of sodium chloride on growth performance, feed utilization, survival rate, and blood components of juvenile Nile tilapia. 


\section{MATERIALS AND METHODS}

\section{Experimental Fish and Culture Technique}

The present study was conducted at the Alfred-Wegener-Institute Helmholtz Center for Polar and Marine Research, Center for Aquaculture Research (ZAF), Bremerhaven, Germany for 10 weeks (70 days, August to October 2018). Two hundred and seventy Nile tilapia, $O$. niloticus juveniles were purchased from Til-Aqua International (The Netherlands; for details on the production see www.til-aqua.com) with an average initial body weight of $8.0 \pm 0.01 \mathrm{~g}$. They were then randomly stocked in 18 glass aquaria $(80 \mathrm{X} 30$ $X 40 \mathrm{~cm}$; length $X$ width $X$ height) each aquaria was stocked with 15 fish. Three different water salinity levels $(0,10$ and $15 \mathrm{ppt})$ and two different dietary sodium chloride $\mathrm{NaCl}$ supplementation levels $(0$ and $10 \%)$ in a factorial manner $(3 \times 2)$ was conducted to represented six different experimental treatments, Three replicates aquaria were randomly assigned to each treatment. Prior to the start of experiment, the fish were acclimated to the experimental conditions for two weeks.

\section{Experimental Diets}

Two experimental diets were formulated to be isonitrogenous, $35 \%$ crude protein $(\mathrm{CP})$ and isocaloric, $10.26 \mathrm{MJ} / \mathrm{kg}$ digestible energy (DE). All diets were identical except for the variation in $\mathrm{NaCl}$ levels. The basal experimental diet had no $\mathrm{NaCl}$ added. (Table 1), Diets 2 contained sodium chloride, $\mathrm{NaCl}$ at levels $10 \%$.

The diets were processed by blending the dry ingredients into a homogenous mixture. Pellets of $2 \mathrm{~mm}$ were made in ZAF laboratory pellet. The pelleting temperature did not exceed $40{ }^{\circ} \mathrm{C}$ and all diets were air dried for $4 \mathrm{hr}$., (moisture content of about 10\%). All diets was packed in cellophane bags and cooled at $4^{\circ} \mathrm{C}$ prior to use.

Fish were fed the experimental diets at the rate of 3\% of body weight per day and it offered two feedings at 8.00 and 13.00 hours. The fish in each aquarium were weighed every 2 weeks, and the feed weight was adjusted after each fish weighing.

Aeration of the water was continuously provided using compressed air.Salinity, temperature, dissolved oxygen, $\mathrm{pH}$ and ammonia were continuously monitored during the experiment as a normal routine for RAS to maintain water quality at optimum range for Nile tilapia. 
Table 1. Formulation and proximate composition of the experimental basal diet (\% as-fed basis)

Ingredients

Percent in basal experimental diet

Fish meal

30

Soybean meal

20

Yellow corn

Wheat bran

Sunflower oil

Vitamin and Mineral premix ${ }^{a}$

Proximate composition (\%)

Dry matter

Crude protein

Crude fat

Total carbohydrate

47.36

Ash

9.93

Gross energy $^{\mathrm{b}}(\mathbf{M J} / \mathbf{k g})$

10.26

a Vitamin and mineral mixture (supplements per kg of the mixed feed): Vit. A 72000IU, Vit. B1 6 mg, Vit. B3 12000 IU, Vit. B6 9 mg, B12 0.06 mg, Vit E 60 mg, Vit. 12 mg, Pantothonic acid $60 \mathrm{mg}$, Nicotinic acid $120 \mathrm{mg}$, Folic acid $6 \mathrm{mg}$, Biotin $0.3 \mathrm{mg}$ and Choline chlorids $3 \mathrm{mg}$.

Each one $\mathrm{Kg}$ of mineral mixture contained: Zinc sulphat hepahydrate $3.0, \mathrm{Mg}$, sulphat 0.335, Coppous chloride 0.10, Calcium phosphate monobasic 135.8, Calcium Lactate 327.0, Ferric citrate 29.7, Potassium phosphate dibasic anhydrous 239.8, Sodium phosphate monobasic 87.2, Sodium chloride 43.6, Aluminium chloride anhydrous 0.15 , Potassium iodide 0.15, Cobalt chloride 1.0, Sodium selenite 0.011 and L-cellulose 132.25 ( as $\mathrm{g} / \mathrm{Kg}$ mineral mix) (Gatlin and Wilson 1984).

${ }^{\mathrm{b}}$ Calculated using gross caloric values of $23.62,39.52$, and $17.15 \mathrm{~kJ} / \mathrm{g}$ for protein, fat, and carbohydrate, respectively, according to Brett (1973).

\section{Growth Indices}

Mean final body weight (FBW) of each experimental treatment was determined by dividing total fish weight in each pen by number of fish. Weight gain (WG), specific growth rate (SGR), feed conversion ratio (FCR), were calculated using the following equations: 
$\mathrm{WG}=$ Final body weight $(\mathrm{g})$ - Initial body weight $(\mathrm{g})$; SGR $=(\ln \mathrm{FBW}-\ln \mathrm{IBW}) / \mathrm{t} \times 100$; where: FBW is final body weight $(\mathrm{g})$; IBW is initial body weight $(\mathrm{g})$; $\mathrm{ln}=$ natural logarithmic; $\mathrm{t}=$ time in days; FCR $=$ Feed intake $(\mathrm{g}) /$ weight gain $(\mathrm{g})$.

\section{Blood Samples and Analysis}

Blood samples were collected at the end of the experiment. Each of the experimental treatment was sampled once for hematological indices analyses. The fish were euthanized with MS-222 and the blood samples were taken by puncturing the caudal vessels. Heparin was used as anticoagulant and the plasma separated by centrifugation at 3000 $\mathrm{rpm}$ for $20 \mathrm{~min}$ and stored at $-20^{\circ} \mathrm{C}$ until further analysis.

\section{Analytical Methods}

At the beginning of the trial, a random pooled sample of 10 fish was collected, for determination of initial whole-body proximate composition. At the termination of the feeding trial, five fish were randomly selected from each pen, homogenized in a blender, to determine the final whole-body proximate composition. The fish were pooled for each pen, oven-dried, ground, and stored at $-20^{\circ} \mathrm{C}$ for subsequent analysis. The chemical composition of the whole fish body and diet was determined according to the procedure of AOAC, (1995). Dry matter was determined after drying the samples in an oven $\left(105^{\circ} \mathrm{C}\right)$ for $24 \mathrm{hr}$. Ash by incineration at $550^{\circ} \mathrm{C}$ for $12 \mathrm{hr}$. Crude protein was determined by micro-Kjeldhal method, $\mathrm{N} \% \times 6.25$ crude fat by soxhlet extraction with diethyl ether $\left(40-60^{\circ} \mathrm{C}\right)$.

\section{Statistical Analysis}

Data of the experiments were analyzed by two-way analysis of variance ANOVA. Significant differences were considered at $\mathrm{P}<0.05$. When significant differences were found,Duncan's multiple range tests was used to identify differences among experimental groups. All statistical analyses were performed using Duncan multiple range test at (P0.05) level SPSS, (1997).

\section{RESULTS}

Final body weight, specific growth rate and weight gain were significantly affected by $\mathrm{NaCl}$ supplementation, water salinity and their interaction $(\mathrm{P}<0.05$; Table 2$)$. Fish fed diets supplemented with $10 \% \mathrm{NaCl}$ recorded significantly higher final body weight when compared to fish fed control either $10(\mathrm{p}<0.05)$ or $15 \mathrm{ppt}(\mathrm{p} \geq 0.05)$ salinity water over a period of ten weeks. Fish fed diets supplemented with $10 \% \mathrm{NaCl}$ recorded significantly higher weight gain when compared to fish fed control either $10 \mathrm{ppt}(\mathrm{p}<0.05)$ and $15 \mathrm{ppt}$ $(\mathrm{p}<0.05)$ salinity water over all experimental period. 
Table (2) Growth performance of Nile tilapia juveniles at different experimental treatments

\section{Experimental treatments}

\begin{tabular}{|c|c|c|c|c|c|c|c|}
\hline \multirow{2}{*}{$\begin{array}{l}\begin{array}{l}\text { Dietary } \\
\text { levels }\end{array} \\
\text { Water salinity } \\
\text { levels, ppt }\end{array}$} & \multicolumn{3}{|c|}{$\mathbf{D}_{\mathbf{0} \%}$} & \multicolumn{3}{|c|}{$\mathbf{D}_{10 \%}$} & \multirow[t]{2}{*}{$P$ value } \\
\hline & $\mathrm{W}_{0 p p t}$ & $\mathrm{~W}_{10 \mathrm{ppt}}$ & $\mathrm{W}_{15 p p t}$ & $\mathrm{~W}_{\text {0ppt }}$ & $\mathrm{W}_{10 \mathrm{ppt}}$ & $\mathrm{W}_{15 p p t}$ & \\
\hline Initial weight (g) & $8.00 \pm 0.01$ & $8.00 \pm 0.01$ & $8.00 \pm 0.01$ & $8.00 \pm 0.01$ & $8.00 \pm 0.01$ & $8.00 \pm 0.01$ & 0.561 \\
\hline $\begin{array}{l}\text { Final body } \\
\text { weight }(g)\end{array}$ & $43.00 \pm 0.68^{\mathrm{a}}$ & $36.00 \pm 1.30^{\mathrm{cd}}$ & $33.75 \pm 0.75^{\mathrm{d}}$ & $41.80 \pm 0.36^{\mathrm{a}}$ & $38.57 \pm 0.22^{b}$ & $36.90 \pm 0.95^{\mathrm{bc}}$ & 0.024 \\
\hline $\begin{array}{l}\text { Specific growth } \\
\text { rate }(\% / \text { day })\end{array}$ & $1.04 \pm 0.01^{\mathrm{a}}$ & $0.94 \pm 0.03^{b}$ & $0.90 \pm 0.02^{\mathrm{c}}$ & $1.03 \pm 0.01^{\mathrm{a}}$ & $0.97 \pm 0.01^{b}$ & $0.95 \pm 0.02^{b}$ & 0.036 \\
\hline \multirow[t]{3}{*}{$\begin{array}{l}\text { weight gain } \\
\text { (g/fish) }\end{array}$} & $35.00 \pm 0.68^{\mathrm{a}}$ & $28.00 \pm 1.30^{\mathrm{cd}}$ & $25.75 \pm 0.75^{d}$ & $33.80 \pm 0.36^{\mathrm{a}}$ & $30.57 \pm 0.22^{b}$ & $28.90 \pm 0.95^{\mathrm{bc}}$ & 0.024 \\
\hline & \multicolumn{3}{|c|}{$\begin{array}{c}\text { Effect of dietary } \mathrm{NaCl} \text { levels irrespective of } \\
\text { water salinity levels on growth performance } \\
\text { indices }\end{array}$} & \multicolumn{4}{|c|}{$\begin{array}{c}\text { Effect of water salinity levels irrespective of dietary } \mathrm{NaCl} \\
\text { levels on growth performance indices }\end{array}$} \\
\hline & $\mathbf{D}_{0 \%}$ & $\mathbf{D}_{10 \%}$ & $P$ value & $\mathbf{W}_{\text {0ppt }}$ & $\mathrm{W}_{10 \mathrm{ppt}}$ & $\mathrm{W}_{15 \mathrm{ppt}}$ & $P$ value \\
\hline Initial weight (g) & $8.00 \pm 0.01$ & $8.00 \pm 0.01$ & 0.650 & $8.00 \pm 0.01$ & $8.00 \pm 0.01$ & $8.00 \pm 0.01$ & 0.750 \\
\hline $\begin{array}{l}\text { Final body } \\
\text { weight }(g)\end{array}$ & $37.58 \pm 1.33^{b}$ & $39.09 \pm 0.78^{\mathrm{a}}$ & 0.031 & $42.40 \pm 0.44^{\mathrm{a}}$ & $37.29 \pm 0.63^{b}$ & $35.33 \pm 0.82^{\mathrm{c}}$ & 0.0001 \\
\hline $\begin{array}{l}\text { Specific growth } \\
\text { rate }(\% / \text { day })\end{array}$ & $0.96 \pm 0.02^{\mathrm{b}}$ & $0.98 \pm 0.01^{\mathrm{a}}$ & 0.033 & $1.04 \pm 0.01^{\mathrm{a}}$ & $0.96 \pm 0.01^{\mathrm{b}}$ & $0.93 \pm 0.02^{c}$ & 0.0001 \\
\hline $\begin{array}{l}\text { weight gain } \\
\text { (g/fish) }\end{array}$ & $30.17 \pm 1.33^{b}$ & $31.09 \pm 0.78^{\mathrm{a}}$ & 0.031 & $34.40 \pm 0.44^{\mathrm{a}}$ & $29.62 \pm 0.63^{b}$ & $27.867 \pm 0.34^{\mathrm{c}}$ & 0.0001 \\
\hline
\end{tabular}

No significant effect of either dietary $\mathrm{NaCl}$ supplementation or water salinity and their interaction were observed on feed intake and feed conversion ratio. Irrespective of dietary $\mathrm{NaCl}$ levels, feed intake and feed conversion ratio affected significantly with water salinity $(\mathrm{P}<0.05$; Table3). Feed intake decreased with increasing water salinity. No mortalities were recorded during 10 weeks .Survival (\%) not affected with either dietary $\mathrm{NaCl}$ supplementations or water salinity and their interaction. Fish groups recorded the highest survival (\%) (Table 3) 
Table (3) Feed utilization of Nile tilapia juveniles at different experimental treatments

\begin{tabular}{|c|c|c|c|c|c|c|c|}
\hline \multirow{2}{*}{$\begin{array}{l}\text { Dietary NaCl levels } \\
\text { Water salinity } \\
\text { levels, ppt }\end{array}$} & \multicolumn{3}{|c|}{$\mathbf{D}_{0 \%}$} & \multicolumn{3}{|c|}{$\mathrm{D}_{10 \%}$} & \multirow[t]{2}{*}{$P$ value } \\
\hline & $\mathrm{W}_{\text {0ppt }}$ & $\mathrm{W}_{10 \mathrm{ppt}}$ & $\mathrm{W}_{15 \mathrm{ppt}}$ & $\mathrm{W}_{\text {0ppt }}$ & $\mathrm{W}_{10 \mathrm{ppt}}$ & $\mathrm{W}_{15 \mathrm{ppt}}$ & \\
\hline Feed Intake & $\begin{array}{c}42.91 \pm 0.52 \\
\mathrm{a}\end{array}$ & $41.47 \pm 0.06^{\mathrm{ab}}$ & $40.17 \pm 0.21^{\mathrm{b}}$ & $43.06 \pm 0.11^{\mathrm{a}}$ & $41.06 \pm 0.77^{b}$ & $39.74 \pm 0.59^{b}$ & 0.820 \\
\hline \multirow[t]{3}{*}{$\begin{array}{l}\text { Feed conversion ( } \mathrm{g} \\
\text { feed/g gain) }\end{array}$} & $1.30 \pm 0.02^{\mathrm{d}}$ & $1.49 \pm 0.07^{\mathrm{ab}}$ & $1.56 \pm 0.04^{\mathrm{a}}$ & $1.33 \pm 0.01^{\mathrm{cd}}$ & $1.39 \pm 0.02^{\mathrm{bcd}}$ & $1.46 \pm 0.07^{\mathrm{abc}}$ & 0.249 \\
\hline & \multicolumn{3}{|c|}{$\begin{array}{c}\text { Effect of dietary } \mathrm{NaCl} \text { levels } \\
\text { irrespective of water salinity levels on } \\
\text { Feed utilization }\end{array}$} & \multicolumn{4}{|c|}{$\begin{array}{c}\text { Effect of water salinity levels irrespective of dietary } \\
\mathrm{NaCl} \text { levels on Feed utilization }\end{array}$} \\
\hline & $\mathbf{D}_{0 \%}$ & $D_{10 \%}$ & $P$ value & $\mathbf{W}_{\text {0ppt }}$ & $W_{10 p p t}$ & $W_{15 p p t}$ & $P$ value \\
\hline Feed Intake & $41.52 \pm 0.4$ & $41.29 \pm 0.56$ & 0.613 & $42.99 \pm 0.24^{\mathrm{a}}$ & $41.26 \pm 0.36^{b}$ & $39.96 \pm 0.01^{\mathrm{c}}$ & 0.0001 \\
\hline $\begin{array}{l}\text { Feed conversion (g } \\
\text { feed/g gain) }\end{array}$ & $1.45 \pm 0.04^{\mathrm{a}}$ & $1.39 \pm 0.03^{\mathrm{b}}$ & 0.149 & $1.32 \pm 0.01^{\mathrm{c}}$ & $1.44 \pm 0.03^{\mathrm{b}}$ & $1.51 \pm 0.04^{\mathrm{a}}$ & 0.002 \\
\hline
\end{tabular}

Irrespective of dietary $\mathrm{NaCl}$ supplementation, body moisture and ash contents showed significantly affect with different water salinity $(\mathrm{P}<0.05$; Table4). No significant affect with dietary $\mathrm{NaCl}$ supplementation and their interaction with different water salinity, on body ether extract and ash content was recorded. Ash content insignificantly affected with dietary $\mathrm{NaCl}$ supplementation. Body crude protein content not significantly affected with either dietary $\mathrm{NaCl}$ supplementation or water salinity $(\mathrm{P}<0.05$; Table 4$)$.

Irrespective of water salinity, the blood serum concentrations of $\mathrm{Na}^{+}$has affected significantly $(\mathrm{P}<0.05)$ with dietary $\mathrm{NaCl}$ supplementation. Fish group fed diet containing $10 \% \mathrm{NaCl}$ recorded the highest values of $\mathrm{Na}^{+}$concentration compared to other experimental groups (Table 5). The concentrations of $\mathrm{K}^{+}$in blood serum showed no significant differences either dietary $\mathrm{NaCl}$ supplementation or water salinity levels or their interaction $\left(\mathrm{P}<0.05\right.$; Table 5). The concentrations of $\mathrm{Cl}^{-}$in blood serum affected significantly $(\mathrm{P}<0.05)$ water salinity and their interaction but did not differ significantly with $\mathrm{NaCl}$ supplementation

The concentrations of Glucose in blood serum affected significantly $(\mathrm{P}<0.05)$ water salinity but did not differ significantly with $\mathrm{NaCl}$ supplementation and their interaction. The concentrations of Lactate in blood serum showed no significant differences with dietary $\mathrm{NaCl}$ supplementation, water salinity and their interaction $(\mathrm{P}<0.05$; Table 5). 
Table (4) Proximate Body composition at different experimental treatments

\begin{tabular}{|c|c|c|c|c|c|c|c|}
\hline \multicolumn{8}{|c|}{ Experimental treatments } \\
\hline $\begin{array}{l}\text { Dietary } \mathrm{NaCl} \\
\text { levels }\end{array}$ & & $\mathbf{D}_{0 \%}$ & & & $\mathbf{D}_{10 \%}$ & & $P$ value \\
\hline $\begin{array}{l}\text { Water salinity } \\
\text { levels, ppt }\end{array}$ & $\mathbf{W}_{0 p p t}$ & $\mathrm{~W}_{10 \mathrm{ppt}}$ & $\mathrm{W}_{15 \mathrm{ppt}}$ & $\mathbf{W}_{0 p p t}$ & $\mathrm{~W}_{10 \mathrm{ppt}}$ & $\mathbf{W}_{15 p p t}$ & \\
\hline Moisture (\%) & $70.43 \pm 0.34$ & $71.80 \pm 1.56$ & $71.78 \pm 0.32$ & $70.19 \pm 0.26$ & $72.46 \pm 0.13$ & $72.26 \pm 0.30$ & 0.79 \\
\hline $\begin{array}{l}\text { Ether extract } \\
(\%)\end{array}$ & $19.47 \pm 0.14^{\mathrm{a}}$ & $18.75 \pm 0.26^{\mathrm{ab}}$ & $18.82 \pm 0.30^{\mathrm{ab}}$ & $19.01 \pm 0.39^{\mathrm{ab}}$ & $18.71 \pm 0.23^{\mathrm{ab}}$ & $18.44 \pm 0.10^{\mathrm{b}}$ & 0.682 \\
\hline $\begin{array}{l}\text { Crude protein } \\
(\%)\end{array}$ & $64.20 \pm 0.21^{\mathrm{a}}$ & $62.87 \pm 0.07^{b}$ & $61.57 \pm 0.23^{d}$ & $62.50 \pm 0.45^{\mathrm{bc}}$ & $61.90 \pm 0.32^{\mathrm{cd}}$ & $61.83 \pm 0.09^{\mathrm{cd}}$ & 0.035 \\
\hline \multirow[t]{3}{*}{$\operatorname{Ash}(\%)$} & $14.23 \pm 0.06^{b}$ & $14.32 \pm 0.01^{\mathrm{ab}}$ & $14.62 \pm 0.22^{\mathrm{a}}$ & $14.53 \pm 0.05^{\mathrm{ab}}$ & $14.44 \pm 0.14^{\mathrm{ab}}$ & $14.28 \pm 0.09^{\mathrm{ab}}$ & 0.009 \\
\hline & \multicolumn{3}{|c|}{$\begin{array}{l}\text { Effect of dietary } \mathrm{NaCl} \text { levels irrespective of } \\
\text { water salinity levels on Body composition }\end{array}$} & \multicolumn{4}{|c|}{$\begin{array}{c}\text { Effect of water salinity levels irrespective of dietary } \mathrm{NaCl} \\
\text { levels on Body composition }\end{array}$} \\
\hline & $\mathbf{D}_{0 \%}$ & $\mathbf{D}_{10 \%}$ & $P$ value & $\mathbf{W}_{\text {0ppt }}$ & $\mathrm{W}_{10 \mathrm{ppt}}$ & $\mathbf{W}_{15 p p t}$ & $P$ value \\
\hline Moisture (\%) & $71.34 \pm 0.52$ & $71.63 \pm 0.38$ & 0.606 & $70.31 \pm 0.72$ & $72.13 \pm 0.72$ & $72.02 \pm 0.22$ & 0.036 \\
\hline $\begin{array}{l}\text { Ether extract } \\
(\%)\end{array}$ & $19.01 \pm 0.17^{\mathrm{a}}$ & $18.72 \pm 0.16^{b}$ & 0.182 & $19.24 \pm 0.21^{\mathrm{a}}$ & $18.73 \pm 0.16^{\mathrm{b}}$ & $18.63 \pm 0.17^{\mathrm{b}}$ & 0.074 \\
\hline $\begin{array}{l}\text { Crude protein } \\
(\%)\end{array}$ & $62.88 \pm 0.39$ & $62.08 \pm 0.19$ & 0.801 & $63.35 \pm 0.01$ & $62.38 \pm 0.01$ & $61.70 \pm 0.13$ & 0.770 \\
\hline $\operatorname{Ash}(\%)$ & $14.39 \pm 0.09$ & $14.41 \pm 0.06$ & 0.803 & $14.38 \pm 0.08$ & $14.38 \pm 0.07$ & $14.45 \pm 0.13$ & 0.801 \\
\hline
\end{tabular}

Table (5) The concentrations of $\mathrm{Na}^{+}, \mathrm{K}+, \mathrm{Cl}-$, Glucose, $\mathrm{PH}$ and Lactate in blood serum of Nile tilapia juveniles at different experimental treatments

\begin{tabular}{|c|c|c|c|c|c|c|c|}
\hline \multicolumn{8}{|c|}{ Experimental treatments } \\
\hline $\begin{array}{l}\text { Dietary } \mathrm{NaCl} \\
\text { levels }\end{array}$ & & $\mathrm{D}_{0 \%}$ & & & $\mathrm{D}_{10 \%}$ & & $P$ value \\
\hline $\begin{array}{l}\text { Water salinity } \\
\text { levels, ppt }\end{array}$ & $\mathrm{W}_{\text {0ppt }}$ & $\mathrm{W}_{10 p p t}$ & $\mathrm{~W}_{15 p p t}$ & $\mathrm{~W}_{\text {0ppt }}$ & $\mathrm{W}_{10 \mathrm{ppt}}$ & $\mathrm{W}_{15 p p t}$ & \\
\hline $\mathrm{Na}^{+}$ & $155.33 \pm 2.00^{\mathrm{b}}$ & $165.00 \pm 1.58^{\mathrm{ab}}$ & $164.22 \pm 0.59^{\mathrm{ab}}$ & $165.55 \pm 1.31^{\mathrm{ab}}$ & $166.56 \pm 2.28^{\mathrm{ab}}$ & $172.48 \pm 7.62^{\mathrm{a}}$ & 0.438 \\
\hline $\mathrm{K}^{+}$ & $6.76 \pm 0.31$ & $5.29 \pm 0.61$ & $6.22 \pm 0.57$ & $4.76 \pm 0.54$ & $5.12 \pm 0.91$ & $5.52 \pm 0.59$ & 0.343 \\
\hline $\mathrm{Cl}^{-1}$ & $135.00 \pm 1.86^{\mathrm{b}}$ & $150.45 \pm 0.91^{\mathrm{a}}$ & $153.89 \pm 3.74^{\mathrm{a}}$ & $145.22 \pm 2.23^{\mathrm{a}}$ & $148.33 \pm 1.35^{\mathrm{a}}$ & $146.44 \pm 3.90^{\mathrm{a}}$ & 0.015 \\
\hline Glucose & $57.44 \pm 7.16^{b}$ & $81.44 \pm 4.70^{\mathrm{ab}}$ & $97.78 \pm 2.62^{\mathrm{a}}$ & $68.11 \pm 4.23^{b}$ & $93.00 \pm 26.18^{\mathrm{ab}}$ & $106.33 \pm 8.21^{\mathrm{a}}$ & 0.992 \\
\hline $\mathrm{PH}$ & $7.66 \pm 0.09^{\mathrm{a}}$ & $7.39 \pm 0.04^{b}$ & $7.40 \pm 0.13^{b}$ & $7.56 \pm 0.01^{\mathrm{ab}}$ & $7.43 \pm 0.07^{\mathrm{ab}}$ & $7.48 \pm 0.05^{\mathrm{ab}}$ & 0.462 \\
\hline
\end{tabular}




\begin{tabular}{|c|c|c|c|c|c|c|c|}
\hline Lactat & $1.62 \pm 0.47$ & $2.01 \pm 0.65$ & $2.90 \pm 0.35$ & $2.68 \pm 1.10$ & $3.14 \pm 0.10$ & $3.60 \pm 0.31$ & 0.927 \\
\hline & \multicolumn{3}{|c|}{$\begin{array}{l}\text { Effect of dietary } \mathrm{NaCl} \text { levels irrespective of } \\
\text { water salinity levels on } \mathrm{Na}^{+}, \mathrm{K}+, \mathrm{Cl}- \\
\text { Glucose }, \mathrm{PH} \text { and Lactate concentrations }\end{array}$} & \multicolumn{4}{|c|}{$\begin{array}{c}\text { Effect of water salinity levels irrespective of dietary } \mathrm{NaCl} \text { level } \\
\text { on } \mathrm{Na}+, \mathrm{K}+, \mathrm{Cl}-, \text { Glucose }, \mathrm{PH} \text { and Lactate concentrations }\end{array}$} \\
\hline & $\mathrm{D}_{0 \%}$ & $\mathrm{D}_{10 \%}$ & $P$ value & $\mathrm{W}_{\text {0ppt }}$ & $\mathrm{W}_{10 \mathrm{ppt}}$ & $\mathrm{W}_{15 \mathrm{ppt}}$ & $P$ value \\
\hline $\mathrm{Na}^{+}$ & $161.52 \pm 1.73^{b}$ & $168.20 \pm 2.59^{\mathrm{a}}$ & 0.033 & $160.44 \pm 2.52^{\mathrm{c}}$ & $165.78 \pm 1.29^{b}$ & $168.35 \pm 3.92^{\mathrm{a}}$ & 0.100 \\
\hline $\mathbf{K}^{+}$ & $6.09 \pm 0.34^{\mathrm{a}}$ & $5.13 \pm 0.37^{b}$ & 0.081 & $5.76 \pm 0.526$ & $5.21 \pm 0.49$ & $5.87 \pm 0.40$ & 0.529 \\
\hline $\mathrm{Cl}^{-1}$ & $146.45 \pm 3.16$ & $146.67 \pm 1.43$ & 0.918 & $140.11 \pm 2.627^{c}$ & $149.39 \pm 0.87^{\mathrm{ab}}$ & $150.17 \pm 2.95^{\mathrm{a}}$ & 0.004 \\
\hline Glucose & $78.89 \pm 6.40^{b}$ & $89.15 \pm 9.78^{a}$ & 0.312 & $62.78 \pm 4.419^{\mathrm{c}}$ & $87.22 \pm 12.17^{b}$ & $102.06 \pm 4.30^{\mathrm{a}}$ & 0.020 \\
\hline PH & $7.49 \pm 0.07$ & $7.49 \pm 0.03$ & 0.957 & $7.61 \pm 0.045$ & $7.41 \pm 0.04$ & $7.44 \pm 0.07$ & 0.039 \\
\hline Lactat & $2.18 \pm 0.32^{b}$ & $3.14 \pm 0.36^{\mathrm{a}}$ & 0.068 & $2.15 \pm 0.584^{b}$ & $2.58 \pm 0.39^{b}$ & $3.25 \pm 0.26^{\mathrm{a}}$ & 0.211 \\
\hline
\end{tabular}

Table (6) The Viscero-somatic index (VSI), Hepato-somatic index (HSI), Spleno-somatic index (SSI) and Heart of Nile tilapia juveniles at different experimental treatments

\begin{tabular}{|c|c|c|c|c|c|c|c|}
\hline \multicolumn{8}{|c|}{ Experimental treatments } \\
\hline $\begin{array}{l}\text { Dietary } \mathrm{NaCl} \\
\text { levels }\end{array}$ & & $\mathbf{D}_{\mathbf{0} \%}$ & & & $\mathbf{D}_{10 \%}$ & & $P$ value \\
\hline $\begin{array}{l}\text { Water salinity } \\
\text { levels, \%o }\end{array}$ & $\mathbf{W}_{\text {0ppt }}$ & $\mathrm{W}_{10 \mathrm{ppt}}$ & $W_{15 p p t}$ & $\mathbf{W}_{\mathbf{0 p p t}}$ & $\mathrm{W}_{10 \mathrm{ppt}}$ & $\mathrm{W}_{15 \mathrm{ppt}}$ & \\
\hline VSI & $4.60 \pm 1.06$ & $4.21 \pm 0.91$ & $4.06 \pm 0.29$ & $4.28 \pm 0.83$ & $4.01 \pm 0.36$ & $3.43 \pm 0.58$ & 0.957 \\
\hline HSI & $0.61 \pm 0.11$ & $0.64 \pm 0.13$ & $0.65 \pm 0.11$ & $0.79 \pm 0.17$ & $0.79 \pm 0.12$ & $0.62 \pm 0.01$ & 0.683 \\
\hline SSI & $0.07 \pm 0.01$ & $0.06 \pm 0.02$ & $0.04 \pm 0.01$ & $0.05 \pm 0.02$ & $0.07 \pm 0.01$ & $0.06 \pm 0.01$ & 0.290 \\
\hline \multirow[t]{3}{*}{ Heart } & $0.12 \pm 0.02$ & $0.09 \pm 0.03$ & $0.09 \pm 0.02$ & $0.10 \pm 0.02$ & $0.13 \pm 0.01$ & $0.08 \pm 0.02$ & 0.354 \\
\hline & \multicolumn{3}{|c|}{$\begin{array}{l}\text { Effect of dietary NaCl levels } \\
\text { irrespective of water salinity levels } \\
\text { on VSI , HSI , SSI and Heart }\end{array}$} & \multicolumn{4}{|c|}{$\begin{array}{l}\text { Effect of water salinity levels irrespective of dietary } \\
\text { NaCl levels on VSI , HSI , SSI and Heart }\end{array}$} \\
\hline & $\mathbf{D}_{\mathbf{0} \%}$ & $\mathbf{D}_{10 \%}$ & $P$ value & $\mathbf{W}_{\text {0ppt }}$ & $\mathrm{W}_{10 \mathrm{ppt}}$ & $W_{15 p p t}$ & $P$ value \\
\hline VSI & $4.29 \pm 0.42$ & $3.90 \pm 0.33$ & 0.527 & $4.44 \pm 0.61$ & $4.11 \pm 0.44$ & $3.74 \pm 0.32$ & 0.644 \\
\hline HSI & $0.63 \pm 0.06$ & $0.74 \pm 0.08$ & 0.355 & $0.70 \pm 0.10$ & $0.71 \pm 0.08$ & $0.64 \pm 0.09$ & 0.819 \\
\hline SSI & $0.05 \pm 0.01$ & $0.06 \pm 0.01$ & 0.312 & $0.06 \pm 0.01$ & $0.06 \pm 0.01$ & $0.05 \pm 0.01$ & 0469 \\
\hline Heart & $0.10 \pm 0.01$ & $0.12 \pm 0.01$ & 0.762 & $0.11 \pm 0.01$ & $0.11 \pm 0.02$ & $0.09 \pm 0.01$ & 0.388 \\
\hline
\end{tabular}


The Viscero-somatic index (VSI), Hepato-somatic index (HSI) and Splenosomatic index (SSI) insignificantly affected with dietary $\mathrm{NaCl}$ supplementation, water salinity and their interaction (Table 6).

\section{DISCUSSION}

The salinity of the culture system is known to influence metabolism and homeostatic processes in fish, meaning the organism needs for nutrients and protein particularly, may differ in freshwater and saltwater systems (Altinok and Gizzle 2001). One strategy for increasing tolerance to adverse environments and maintaining growth rates in many species is a more efficient management of diet nutrient contents.

Since minerals absorbed from the water do not always meet the total metabolic requirements in fish, their supplementation through the diet promotes growth Hepher, (1988). Fish diet is therefore an important source of salts not only to satisfy the needs for growth, but also for osmoregulation. Providing a sufficient amount of salt through feeds can spare energy that is used for osmoregulation, thereby reducing stress and allowing more energy for growth. Dietary salt has been found to be beneficial for growth in rainbow trout MacLeod, (1978), common carp and mrigal Nandeesha et al., (2000), Asian sea bass Harpaz et al., (2005), European sea bass (Eroldogan, 2003; Eroldogan et al., 2005) and gilthead sea bream (Appelbaum et al., 2008a; Appelbaum and Arockiaraj, 2008b, 2009).

Results of the current study show that fish fed diets supplemented with $10 \% \mathrm{NaCl}$ recorded significantly better final body weight and weight gain when compared to fish fed control when held in 10 and 15 ppt salinity water. Feed intake and feed conversion ratio in the current study decreased with increasing water salinity reported by (Boeuf and Payan, 2001) reviewed the literature on salinity influence on growth in fish and concluded that salinity is also a key factor in controlling growth. They observed that the changes in growth rate that depend on salinity result from an action on metabolic rate, food intake and food conversion. Better growth at intermediate salinities (8-20 ppt) is very often but not systematically, correlated to a lower standard metabolic rate. Florida red tilapia physiologically function more efficiently in brackish and saline waters, with lower feed conversion ratios and faster weight gain than those raised in freshwater Head et al., (1994).

No mortalities were recorded during 10 weeks. Survival (\%) not affected with either dietary $\mathrm{NaCl}$ supplementations or different water salinity. Fish groups recorded the highest survival (\%). Yao et al., (2008) reported that survival of Nile tilapia fingerlings transferred directly to saline water was increased $(84.3 \%$ to $96.8 \%)$ up to $17 \mathrm{ppt}$, but mortalities were significant decreased above that level of salinity (60-70\%). Higher survival (\%) (78 to $81 \%$ ) was achieved by gradual acclimation to salinity of 30 ppt over 
two days. Results of Lim et al., (2006) showed that juvenile Nile tilapia receiving dietary $\mathrm{NaCl}$ feeding regimens, even for a two-week period, exhibited consistently better survival values.

Salt tolerance in tilapia can be improved by optimizing acclimation protocols, adding salt to the diet. Results of the current study show moisture, ether extract and crude protein not significantly affected with $\mathrm{NaCl}$ supplementation. The contrast results were obtained by Keshavanath et al., (2012), who found that carcass composition was not affected as salt addition in fish diets.

To summarize, the results of this study confirm that the control balance of salt either in diet or water within a narrow limit is critical to life in all multicellular organisms, including teleost fishes. Salt tolerance is a term describing the overall fitness, or productivity, of the fish in a saline environment. The results of the present study revealed that juveniles of Nile tilapia has been documented as surviving in salinities up to $15 \mathrm{ppt}$ with acclimation. The results demonstrating the beneficial effect that supplemental dietary salt can have beneficial effects on fish growth.

\section{Acknowledgement}

This work was supported by the Science and Technolgy Development Fund (STDF), under Grant no. 25513, Ministry of Scientific Research, Egypt.

\section{REFERENCES}

AOAC. (1995). Official Methods of Analysis, 15th ed. Association of Official Analytical Chemists, Arlington, VA.

Altinok I. and Gizzle J.M. (2001). Effects of brackish water on growth, feed conversion and energy absorption effciency by juvenile euryhaline and freshwater stenohaline fishes. Journal of Fish Biology 59;42-1152.

Appelbaum.; S and A.J. Arockiaraj. (2008b). Brackish water sea bream success. Fish Farmer Inter., 35(8): 35.

Appelbaum, S and A.J. Arockiaraj. (2009). Cultivation of gilthead sea bream (Sparus aurata L.) in low salinity inland brackish geothermal water. AACL Bioflux, 2 (2): 197203.

Appelbaum, S., A.J.;Arockiaraj and C. Imanraj.(2008a). Promoting the culture of gilthead sea bream (Sparus auratus L.) in low saline inland water: A novel way to farm saltwater fish in freshwater. Fish for the People, 6(1): 40-44.

Appelbaum, S., A.J.; Arockiaraj and C. Imanraj. (2008b). Cultivation of gilthead sea bream (Sparus auratus L.) in low saline inland water of southern part of Israel desert. Aquaculture Asia, 13 (4): 33-36 
Boeuf, G. and P. Payan. (2001). How should salinity influence fish growth? Comparative Biochemistry and Physiology - Part C: Toxicology and Pharmacology, 130: 411-423.

Brett, J.R.(1973). Energy expenditure of Sockeye salmon Oncorhynchus nerka, during sustained performance. J. Fish. Res. Board Can., 30: 1799-1809.

Cnaani, A. and G. Hulata.(2011). Improving salinity tolerance in tilapias: Past experience and future prospects. Isr. J. Aquacult. - Bamidgeh 63, IIC.63.2011.533, 21 pages.

Eroldogan O. T.; Kumlu M., Kır M.; and Kiris G. A.(2005). Enhancement of growth and feed utilization of the European sea bass (Dicentrarchus labrax) fed supplementary dietary salt in freshwater. Aquacult Res 36:361-369. DOI: 10.1111/j.1365- 2109.2004.01211.x

Eroldogan, O.T.(2003). Acclimation of European sea bass (Dicentrarchus labrax) to freshwater and determination of its optimal feeding rates in freshwater. Ph.D. thesis, Univ. Cukurova, Adana, Turkey.

Fineman Kalio, A.S.(1988). Preliminary observations on the effect of salinity on the reproduction and growth of freshwater Nile tilapia, Oreochromis niloticus (L.), cultured in brackishwater ponds. Aquacult. Fish. Manage. 19:313-320.

Gatlin III D.M. and R.P. Wilson .(1984). Studies of the manganese requirement of fingerling channel catfish. Aquaculture, 41: 85-92.

Harpaz, S.; Hakim, Y.; Slossman. T. and Eroldogan, O.T. (2005). Effects of adding salt to the diet of Asian sea bass Latescal cariferreared in fresh or salt water recalculating tanks, on growth and brush border enzyme activity. Aquaculture, 248: 315-324.

Head, W.D.; Zerbi, A. and Watanabe, W.O. (1994). Preliminary observations on the marketability of salt-water cultured Florida red tilapia in Puerto Rico. Journal of the World Aquaculture Society, 25: 432-441.

Hepher, B. (1988). Nutrition of Pond Fishes. Cambridge University Press, Cambridge, UK., pp 388.

Keshavnath, P.; Oishi, C.A.; Leao da Fonseca, F.A.; Affonso, E.G. and Filho, M.P. (2012). Growth Response of Tambaqui (Colossoma macropomum) Fingerlings to Salt (Sodium Chloride) Supplemented Diets. Journal of Fisheries and Aquatic Science, 7: 439-446.

Larumbe-Morán, E.; Hernández-Vergara, M.P.; Olvera-Novoa, M.A. and Pérez Rostro, C.I. (2010). Protein requirements of Nile tilapia (Oreochromis niloticus) fry cultured at different salinities. Aquaculture Research, 41:.1150-1157.

Lim, C.E.; Aksoy, M.; Welker, T.L., and Veverica, K. (2006). Effect of feeding duration of sodium chloride containing diets on growth performance and some osmoregulatory parameters of Nile tilapia (Oreochromis niloticus) after transfer to water of different salinities. Journal of Applied Aquaculture 18: 1-17. 
MacLeod, M.G. (1978). Relationships between dietary sodium chloride, food intake and food conversion in the rainbow trout. J. Fish Biol., 13: 71-78.

Nandeesha, M. C.; Gangadhar, B.; Keshavanath, P. and Varghese, T. J. (2000). Effect of dietary sodium chloride supplementation on growth, biochemical composition and digestive enzyme activity of young Cyprinus carpio (Linn.) and Cirrhinus mrigala (Ham.). Journal of Aquaculture in the Tropics, 15 (2): 135-144.

SPSS. (1997). Statistical package for the social sciences, Versions16, SPSS in Ch, ChiUSA.

Yao, K.; Ouattara, M. and A.F.A. Ahoussi,(2008). Survie du Tilapia du Nil (Oreochromis niloticus) en eaux salées durant un transfert direct et progressif [Survival of the Nile Tilapia (Oreochromis niloticus) in salt water during a direct and progressive transfer]. Livest. Res. Rural Develop. 20: Article \#72. (http://www.lrrd.org/lrrd20/5/yao20072.htm; in French with English abstract). 